\title{
Two New Xanthones from the Stems of Garcinia cowa
}

\author{
Jie SHEN and Jun-Shan YANG* \\ Institute of Medicinal Plant Development, Chinese Academy of Medical Sciences, Peking Union Medical College; Beijing \\ 100094, People's Republic of China. $\quad$ Received July 26, 2005; accepted September 17, 2005
}

Two new xanthones, 1,5,6-trihydroxy-3-methoxy-4-(3-hydroxyl-3-methylbutyl)xanthone (1) and 1,5-dihydroxy-3-methoxy-6', $6^{\prime}$-dimethyl-2H-pyrano( $\left(2^{\prime}, 3^{\prime}: 6,7\right)-4-(3$-methylbut-2-enyl)xanthone (2), have been isolated together with six known xanthones: 1,3,5-trihydroxy-6',6'-dimethyl-2H-pyrano( $\left(2^{\prime}, 3^{\prime}: 6,7\right)$ xanthone (3), dulxanthone A (4), 1,5,6-trihydroxy-3,7-dimethoxyxanthone (5), 1,7-dihydroxyxanthone (6), 1,3,5-trihydroxy-6methoxyxanthone (7), 1,3,6,7-tetrahydroxyxanthone (8), from the stems of Garcinia cowa (Guttiferae).

Key words Garcinia cowa; Guttiferae; xanthone

The plant Garcinia cowa Roxв. is a medium size tree found in Southeast China and Southeast Asia. The fruits are edible and abundant in (-)-hydroxycitric acid, ${ }^{1)}$ and the latex and the bark have some prenylated xanthones which show mainly a $1,3,6,7$ oxygenation pattern. ${ }^{2-6)}$ The constituents of the young stems of the species from Southeast China were investigated for bioactive xanthones. This paper reports the isolation and characterization of two new xanthones, 1,5,6trihydroxy-3-methoxy-4-(3-hydroxyl-3-methylbutyl)xanthone (1) and 1,5-dihydroxy-3-methoxy-6', $6^{\prime}$-dimethyl- $2 \mathrm{H}$ pyrano $\left(2^{\prime}, 3^{\prime}: 6,7\right)-4-(3-m e t h y l b u t-2-e n y l) x a n t h o n e ~(2)$, along with six known xanthones: 1,3,5-trihydroxy-6',6'-dimethyl$2 H$-pyrano $\left(2^{\prime}, 3^{\prime}: 6,7\right)$ xanthone (3), ${ }^{7)}$ dulxanthone A (4) ${ }^{8)}$ 1,5,6-trihydroxy-3,7-dimethoxyxanthone (5), ${ }^{9)}$ 1,7-dihydroxyxanthone (6), ${ }^{10)} 1,3,5$-trihydroxy-6-methoxyxanthone (7), ${ }^{11)}$ $1,3,6,7$-tetrahydroxyxanthone $(\mathbf{8}),{ }^{12)}$ from the stems of this plant. Compounds $1-4,7$ have a 1, 3, 5, 6 oxygenation pattern of the xanthone nucleus. Examination of their bioactivities is now in progress.

Compound 1, obtained as a pale yellow amorphous powder, had the molecular formula $\mathrm{C}_{19} \mathrm{H}_{20} \mathrm{O}_{7}$ based on $\mathrm{m} / \mathrm{z}$ $360.1216\left(\mathrm{M}^{+}\right)$by high-resolution electron impact mass spectroscopy (HR-EI-MS). Its UV (234, 252, 285, $326 \mathrm{~nm})$ and IR $\left(3469,1641 \mathrm{~cm}^{-1}\right)$ showed absorptions characteristic of 1,3,5,6-tetraoxygenated xanthone. ${ }^{8,9,13)}$ In the ${ }^{1} \mathrm{H}-\mathrm{NMR}$ spectrum, four hydroxyl signals $\left[\delta_{\mathrm{H}} 4.32,9.06,10.51(1 \mathrm{H}\right.$, each, br s), and $13.11(1 \mathrm{H}, \mathrm{s}$, chelated)] were observed; two one-proton doublets $\left[\delta_{\mathrm{H}} 6.87,7.46\right.$, each $\left.J=9 \mathrm{~Hz}\right]$ were further exhibited in addition to a methoxyl singlet $\left[\delta_{\mathrm{H}} 3.85(3 \mathrm{H}\right.$, s)]. The spectrum also showed the presence of two methyl groups in a singlet $\left[\delta_{\mathrm{H}} 1.12(6 \mathrm{H}, \mathrm{s})\right]$ and two methylene groups as two triplets $\left[\delta_{\mathrm{H}} 1.52,2.77\right.$, each $J=8.5 \mathrm{~Hz}$ ], implying the presence of a 3-hydroxyl-3-methylbutyl side chain.

The ${ }^{13} \mathrm{C}-\mathrm{NMR}$ data of $\mathbf{1}$ (see Table 1) had a strong resemblance to 4 (characterized as dulxanthone A) indicating a xanthone skeleton with a similar substitution pattern. ${ }^{8}$ Furthermore, the ${ }^{1} \mathrm{H}$ - and ${ }^{13} \mathrm{C}-\mathrm{NMR}$ spectroscopic assignments for 1 were determined by the HSQC and HMBC spectrum (see Fig. 2). In the HMBC spectrum, cross-peaks the chelated hydroxyl proton $\delta_{\mathrm{H}} 13.11(1-\mathrm{OH}) / \delta_{\mathrm{C}} 94.1(\mathrm{C}-2)$ and $101.9(\mathrm{C}-9 \mathrm{a}), \delta_{\mathrm{H}} 6.42[\mathrm{H}-2] / \delta_{\mathrm{C}} 160.9(\mathrm{C}-1), 101.9(\mathrm{C}-9 \mathrm{a})$ and $163.4(\mathrm{C}-3), \delta_{\mathrm{H}} 3.85\left(3-\mathrm{OCH}_{3}\right) / \delta_{\mathrm{C}} 163.4(\mathrm{C}-3)$ were observed; correlations $\delta_{\mathrm{H}} 2.77\left[\mathrm{H}-1^{\prime}\right] / \delta_{\mathrm{C}} 163.4(\mathrm{C}-3), 153.5$ (C$4 a)$ and $108.5(\mathrm{C}-4), \delta_{\mathrm{H}} 1.52\left[\mathrm{H}-2^{\prime}\right] / \delta_{\mathrm{C}} 108.5(\mathrm{C}-4)$ were present, which confirmed the A-ring feature of 1 . Furthermore, one hydroxyl group $\delta_{\mathrm{H}} 9.06[5-\mathrm{OH}]$ showed correlations to
$\delta_{\mathrm{C}} 152.2(\mathrm{C}-6), 132.5(\mathrm{C}-5)$ and $146.5(\mathrm{C}-10 \mathrm{a})$, another hydroxyl group $\delta_{\mathrm{H}} 10.51[6-\mathrm{OH}]$ was correlated to $\delta_{\mathrm{C}} 152.2$ $(\mathrm{C}-6), 132.5(\mathrm{C}-5)$ and $112.9(\mathrm{C}-7)$; correlation between $\delta_{\mathrm{H}}$ $7.46[\mathrm{H}-8]$ and $\delta_{\mathrm{C}} 180.8(\mathrm{C}-9)$ was observed, which indicated the position of two hydroxyl groups in the B-ring. Thus, compound 1 was determined to be 1,5,6-trihydroxy-3methoxy-4-(3-hydroxyl-3-methylbutyl)xanthone.

Compound 2 was isolated as yellow needles, mp 217$218^{\circ} \mathrm{C}, \mathrm{C}_{24} \mathrm{H}_{24} \mathrm{O}_{6}(m / z 408.1552)$, had UV and IR spectral data suggestive of a xanthone derivative. What is more, 2 showed analogous UV features to nigrolineaxanthone $\mathrm{B}(1,5-$ dihydroxy-3-methoxy- $6^{\prime}, 6^{\prime}$-dimethyl- $2 H$-pyrano $\left(2^{\prime}, 3^{\prime}: 6,7\right)$ 4-(3-hydroxyl-3-methylbutyl)xanthone) also suggesting the presence of a 1,3,5,6-tetraoxygenated xanthone chromophore. ${ }^{13)}$ In the ${ }^{1} \mathrm{H}-\mathrm{NMR}$ spectrum, two hydroxyl groups [ $\delta_{\mathrm{H}} 9.37(1 \mathrm{H}, \mathrm{brs})$, and $13.14(1 \mathrm{H}, \mathrm{s}$, chelated $\left.)\right]$ were observed; in addition to a methoxyl singlet $\left[\delta_{\mathrm{H}} 3.90\right]$, a set of signals due to a prenyl group $\left[\delta_{\mathrm{H}} 3.46(2 \mathrm{H}, \mathrm{d}, J=7.5 \mathrm{~Hz})\right.$, $5.20(1 \mathrm{H}, \mathrm{t}, J=7.5 \mathrm{~Hz}), 1.79(3 \mathrm{H}, \mathrm{s}), 1.61(3 \mathrm{H}, \mathrm{s})]$ was present; there were two aromatic proton signals at $\delta_{\mathrm{H}} 7.38$ and $6.49($ each $1 \mathrm{H}, \mathrm{s})$, the signal in lower field $\left[\delta_{\mathrm{H}} 7.38\right]$ was assigned to $\mathrm{H}-8$ which was strongly deshielded by the carbonyl, and the other signal at $\delta_{\mathrm{H}} 6.49(1 \mathrm{H}, \mathrm{s})$ was indicative of $\mathrm{H}-2$ or H-4. Two one-proton doublets $\left[\delta_{\mathrm{H}} 6.57,5.88\right.$, each $J=10 \mathrm{~Hz}]$ along with two methyl groups $\left[\delta_{\mathrm{H}} 1.45,6 \mathrm{H}, \mathrm{s}\right]$ suggested the presence of a 2,2-dimethylpyran ring.

Thus, the structure of $\mathbf{2}$ was characterized by the HSQC and HMBC spectrum (see Fig. 2). In the HMBC spectrum (as shown), cross peaks $\delta_{\mathrm{H}} 13.14(1-\mathrm{OH}) / \delta_{\mathrm{C}} 94.3(\mathrm{C}-2)$ and $102.0(\mathrm{C}-9 \mathrm{a}), \delta_{\mathrm{H}} 3.90\left(3-\mathrm{OCH}_{3}\right) / \delta_{\mathrm{C}} 163.6(\mathrm{C}-3)$ were observed; $\delta_{\mathrm{H}} 3.46\left[\mathrm{H}-1^{\prime \prime}\right]$ was correlated to $\delta_{\mathrm{C}} 163.6(\mathrm{C}-3)$, 153.1 (C-4a) and 107.5 (C-4), which confirmed a prenyl group was attached to $\mathrm{C}-4$. Furthermore, the A-ring feature of $\mathbf{2}$ was confirmed by comparison of the ${ }^{13} \mathrm{C}$-NMR data with the isolated compounds $\mathbf{1}$ and $\mathbf{4}$ (see Table 1) including literature values. The presence of a dimethylpyran ring fused with the $\mathrm{B}$ ring of the 1,3,5,6-tetraoxygenated xanthone nucleus at $\mathrm{C}-6$ and $\mathrm{C}-7$ was determined by three $\mathrm{C}-\mathrm{H}$ threebond correlations $\delta_{\mathrm{H}} 5.88\left[\mathrm{H}-5^{\prime}\right] / \delta_{\mathrm{C}} 118.2(\mathrm{C}-7), \delta_{\mathrm{H}} 6.57[\mathrm{H}-$ $\left.4^{\prime}\right] / \delta_{\mathrm{C}} 146.3(\mathrm{C}-6)$ and $112.1(\mathrm{C}-8)$. Since there was no other proton signal the substituent at $\mathrm{C}-5$ must be a hydroxyl group, which was established by $\mathrm{HMBC}$ correlations $\boldsymbol{\delta}_{\mathrm{H}} 9.37$ $[5-\mathrm{OH}] / \delta_{\mathrm{C}} 133.2(\mathrm{C}-5), 146.3(\mathrm{C}-6)$ and $146.3(\mathrm{C}-10 \mathrm{a})$. Accordingly, compound 2 was elucidated as 1,5-dihydroxy-3methoxy-6', $6^{\prime}$-dimethyl-2H-pyrano(2',3':6,7)-4-(3-methylbut-2-enyl)xanthone. 
Table $1 .{ }^{13} \mathrm{C}-\mathrm{NMR}$ Data of Compounds $\mathbf{1}-\mathbf{5}$ (Values in $(\boldsymbol{\delta}) \mathrm{ppm}$ )

\begin{tabular}{|c|c|c|c|c|c|}
\hline & 1 & 2 & 3 & 4 & 5 \\
\hline $\mathrm{C}-1$ & 160.9 & 161.2 & 162.8 & 161.2 & 162.3 \\
\hline $\mathrm{C}-2$ & 94.1 & 94.3 & 97.9 & 94.2 & 96.7 \\
\hline $\mathrm{C}-3$ & 163.4 & 163.6 & 165.2 & 163.5 & 165.6 \\
\hline $3-\mathrm{OCH}_{3}$ & 56.3 & 56.4 & & 56.3 & 55.9 \\
\hline C-4 & 108.5 & 107.5 & 93.9 & 107.1 & 92.3 \\
\hline$C-4 a$ & 153.5 & 153.1 & 157.2 & 153.2 & 157.1 \\
\hline $\mathrm{C}-5$ & 132.5 & 133.2 & 133.0 & 132.6 & 133.3 \\
\hline C-6 & 152.2 & 146.3 & 146.0 & 152.3 & 142.2 \\
\hline$C-7$ & 112.9 & 118.2 & 118.2 & 112.9 & 146.0 \\
\hline C-8 & 116.0 & 112.1 & 112.0 & 116.0 & 95.3 \\
\hline$C-8 \mathrm{a}$ & 112.8 & 113.5 & 113.7 & 112.8 & 111.2 \\
\hline $\mathrm{C}-9$ & 180.8 & 180.1 & 179.4 & 180.2 & 179.4 \\
\hline$C-9 a$ & 101.9 & 102.0 & 101.6 & 101.8 & 102.4 \\
\hline$C-10 a$ & 146.5 & 146.3 & 146.0 & 146.5 & 142.2 \\
\hline \multirow[t]{10}{*}{ Others } & $17.2\left(\mathrm{C}-1^{\prime}\right)$ & $121.1\left(\mathrm{C}-4^{\prime}\right)$ & $121.1\left(\mathrm{C}-4^{\prime}\right)$ & $21.1\left(\mathrm{C}-1^{\prime}\right)$ & $55.9\left(7-\mathrm{OCH}_{3}\right)$ \\
\hline & $42.7\left(\mathrm{C}-2^{\prime}\right)$ & $131.7\left(\mathrm{C}-5^{\prime}\right)$ & $131.6\left(\mathrm{C}-5^{\prime}\right)$ & $122.2\left(\mathrm{C}-2^{\prime}\right)$ & \\
\hline & $69.3\left(\mathrm{C}-3^{\prime}\right)$ & $77.8\left(\mathrm{C}-6^{\prime}\right)$ & $77.6\left(\mathrm{C}-6^{\prime}\right)$ & $130.9\left(\mathrm{C}-3^{\prime}\right)$ & \\
\hline & $29.2\left(\mathrm{C}-4^{\prime}\right)$ & $27.8\left(6^{\prime}-\mathrm{CH}_{3}\right)$ & $27.9\left(6^{\prime}-\mathrm{CH}_{3}\right)$ & $25.5\left(\mathrm{C}-4^{\prime}\right)$ & \\
\hline & $29.2\left(\mathrm{C}-5^{\prime}\right)$ & $27.8\left(6^{\prime}-\mathrm{CH}_{3}\right)$ & $27.9\left(6^{\prime}-\mathrm{CH}_{3}\right)$ & $17.7\left(\mathrm{C}-5^{\prime}\right)$ & \\
\hline & & $21.1\left(\mathrm{C}-1^{\prime \prime}\right)$ & & & \\
\hline & & $122.1\left(\mathrm{C}-2^{\prime \prime}\right)$ & & & \\
\hline & & $131.0\left(\mathrm{C}-3^{\prime \prime}\right)$ & & & \\
\hline & & $25.5\left(\mathrm{C}-4^{\prime \prime}\right)$ & & & \\
\hline & & $17.7\left(\mathrm{C}-5^{\prime \prime}\right)$ & & & \\
\hline
\end{tabular}

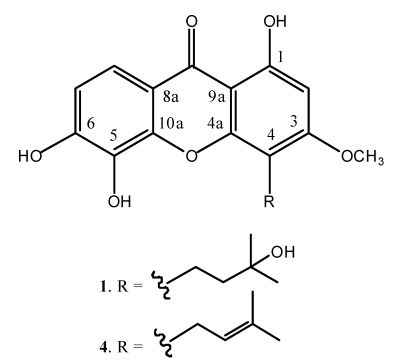<smiles>CC1(C)C=Cc2cc3c(=O)c4c(O)cc(Br)c(Br)c4oc3c(O)c2O1</smiles>

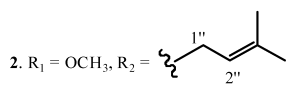
3. $\mathrm{R}_{1}=\mathrm{OH}, \mathrm{R}_{2}=\mathrm{H}$

Fig. 1. Chemical Structures of $1-4$
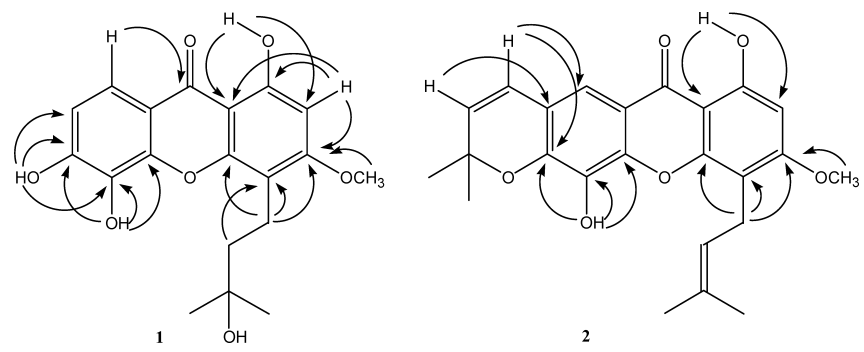

Fig. 2. Selected HMBC Correlations of $\mathbf{1}$ and $\mathbf{2}$

Compounds 3 and 5 were determined as 1,3,5-trihydroxy$6^{\prime}, 6^{\prime}$-dimethyl- $2 H$-pyrano $\left(2^{\prime}, 3^{\prime}: 6,7\right)$ xanthone and 1,5,6-trihydroxy-3,7-dimethoxyxanthone on the basis of the HSQC and $\mathrm{HMBC}$ spectrum involving comparison of their MS and ${ }^{1} \mathrm{H}-\mathrm{NMR}$ data. The ${ }^{13} \mathrm{C}$-NMR spectra of 1,5,6-trihydroxy3,7-dimethoxyxanthone (5) are reported here for the first time.

\section{Experimental}

General Experimental Procedures Melting points were determined on an X4 micro-melting point apparatus and are uncorrected. UV spectra were measured with a Hitachi UV-2201 spectrophotometer and IR spectra with an Impact 400 FTIR spectrometer. ${ }^{1} \mathrm{H}$ - and ${ }^{13} \mathrm{C}$-NMR spectra were recorded in DMSO- $d_{6}$ or $\mathrm{CDCl}_{3}$ with an INOVA spectrometer at $500 \mathrm{MHz}\left({ }^{1} \mathrm{H}\right)$ and
$125 \mathrm{MHz}\left({ }^{13} \mathrm{C}\right)$, using visual DMSO- $d_{6}$ resonances for internal reference. The spectra were interpreted with aid of the HMBC and HSQC techniques. Mass spectra were recorded on an AutoSpec Ultima-TOF spectrometer. Silica gel (200-300 mesh) and silica gel $\mathrm{GF}_{254}$ sheets $(0.20-0.25 \mathrm{~mm})$ (from Qingdao Haiyang Chemical Co., Qingdao, P. R. China) were used for column chromatography and TLC, respectively. Sephadex LH-20 (25$100 \mu \mathrm{m}$, Sigma-Aldrich) was also used for column chromatography.

Plant Material The stems of Garcinia cowa were collected from Yunnan Province P. R. China and identified by Prof. ShaoRong Guo. A voucher specimen (YA-04-0418) is deposited at the Institute of Medicinal Plant Development, Chinese Academy of Medical Sciences and Peking Union Medical College, P. R. China.

Extraction and Isolation The dried stems of Garcinia cowa $(20 \mathrm{~kg})$ were extracted three times with $95 \% \mathrm{EtOH}$ for $2 \mathrm{~h}$ under reflux, and then extracted two times with $70 \% \mathrm{EtOH}$ for $2 \mathrm{~h}$ under reflux. After combination and removal of solvent, the residue $(2.1 \mathrm{~kg})$ was suspended in water $(5000 \mathrm{ml})$ and partitioned successively with petroleum ether $(5000 \mathrm{ml})$, EtOAc $(5000 \mathrm{ml})$, and $n-\mathrm{BuOH}(5000 \mathrm{ml})$. The EtOAc-soluble fraction $(272 \mathrm{~g})$ was chromatographed over silica gel and eluted with $\mathrm{CHCl}_{3}-\mathrm{MeOH}$ $(10: 0-1: 1)$ to give 16 fractions. Fraction $3(1.5 \mathrm{~g})$ was separated by a silica gel (200-300 mesh) column eluting with ether-EtOAc ( $7: 3)$ to afford 2 $(35 \mathrm{mg})$. Fraction $5(61.5 \mathrm{~g})$ was separated by a silica gel $(200-300 \mathrm{mesh})$ column eluting with $\mathrm{CHCl}_{3}-\mathrm{MeOH}(9: 1)$ to afford 1 (200 mg), and on Sephadex LH-20, eluting with $\mathrm{MeOH}$, to afford $3(80 \mathrm{mg})$. Fraction 6 $(11.3 \mathrm{~g})$ was purified by Sephadex $\mathrm{LH}-20$, and elution with $\mathrm{MeOH}-\mathrm{H}_{2} \mathrm{O}$ $(95: 5)$ gave $4(18 \mathrm{mg})$ and 5. Fractions $8-10$ were also separated by Sephadex LH-20 with MeOH : $\mathrm{H}_{2} \mathrm{O}(95: 5)$ to afford $6(110 \mathrm{mg}), 7(23 \mathrm{mg})$, and 8 (300 mg).

1,5,6-Trihydroxy-3-methoxy-4-(3-hydroxyl-3-methylbutyl)xanthone (1): Pale yellow amorphous powder. ${ }^{1} \mathrm{H}-\mathrm{NMR} \delta: 13.11(\mathrm{H}, \mathrm{brs}, 1-\mathrm{OH}), 10.51$ (H, br s, 6-OH), $9.06(\mathrm{H}$, br s, 5-OH), $7.46(\mathrm{H}, \mathrm{d}, J=9 \mathrm{~Hz}, \mathrm{H}-8), 6.87(\mathrm{H}, \mathrm{d}$, $J=9 \mathrm{~Hz}, \mathrm{H}-7), 6.42(\mathrm{H}, \mathrm{s}, \mathrm{H}-2), 4.32\left(\mathrm{H}, \mathrm{br} \mathrm{s}, 3^{\prime}-\mathrm{OH}\right), 3.85\left(3 \mathrm{H}, \mathrm{s}, 3-\mathrm{OCH}_{3}\right)$, $2.77\left(2 \mathrm{H}, \mathrm{t}, J=8.5 \mathrm{~Hz}, \mathrm{H}-1^{\prime}\right), 1.52\left(2 \mathrm{H}, \mathrm{t}, J=8.5 \mathrm{~Hz}, \mathrm{H}-2^{\prime}\right), 1.12\left(6 \mathrm{H}, \mathrm{s}, \mathrm{H}_{3}-\right.$ $4^{\prime}, \mathrm{H}_{3}-5^{\prime}$ ). ${ }^{13} \mathrm{C}-\mathrm{NMR}$ data (see Table 1). IR (KBr) $v_{\max } \mathrm{cm}^{-1}: 3469,3312$, $2960,2820,1641,1585,1466,1432,1365,1262 . \mathrm{UV}\left(\mathrm{CH}_{3} \mathrm{OH}\right) \lambda_{\max }(\log \varepsilon)$ : 234 (4.35), 252 (4.57), 285 (3.89), 326 (4.21). HR-EI-MS $\mathrm{m} / \mathrm{z} 360.1216$ $[\mathrm{M}]^{+}$(Calcd for $\left.\mathrm{C}_{19} \mathrm{H}_{20} \mathrm{O}_{7}: 360.1209\right)$. EI-MS $\mathrm{m} / z 360$ ([M] $\left.]^{+}, 13\right), 342(21)$, 327 (8), 301 (7), 287 (73), 286 (100), 257 (14).

1,5-Dihydroxy-3-methoxy-6', $6^{\prime}$-dimethyl- $2 H$-pyrano $\left(2^{\prime}, 3^{\prime}: 6,7\right)-4-(3-$ methylbut-2-enyl)-xanthone (2): Yellow needles, $\mathrm{mp} 217-218^{\circ} \mathrm{C}\left(\mathrm{CHCl}_{3}\right)$. ${ }^{1} \mathrm{H}-\mathrm{NMR} \delta$ : $13.14(\mathrm{H}$, br s, 1-OH), $9.37(\mathrm{H}, \mathrm{brs}, 5-\mathrm{OH}), 7.38(\mathrm{H}, \mathrm{s}, \mathrm{H}-8)$, $6.57\left(\mathrm{H}, \mathrm{d}, J=10 \mathrm{~Hz}, \mathrm{H}-4^{\prime}\right), 6.49(\mathrm{H}, \mathrm{s}, \mathrm{H}-2), 5.88\left(\mathrm{H}, \mathrm{d}, J=10 \mathrm{~Hz}, \mathrm{H}-5^{\prime}\right)$, $5.20\left(\mathrm{H}, \mathrm{t}, J=7.5 \mathrm{~Hz}, \mathrm{H}-2^{\prime \prime}\right), 3.90\left(3 \mathrm{H}, \mathrm{s}, 3-\mathrm{OCH}_{3}\right), 3.46(2 \mathrm{H}, \mathrm{d}, J=7.5 \mathrm{~Hz}$, 
$\left.\mathrm{H}-1^{\prime \prime}\right), 1.79\left(3 \mathrm{H}, \mathrm{s}, \mathrm{H}-4^{\prime \prime}\right), 1.61\left(3 \mathrm{H}, \mathrm{s}, \mathrm{H}-5^{\prime \prime}\right), 1.45\left(6 \mathrm{H}, \mathrm{s}, 6^{\prime}, 6^{\prime}-\mathrm{CH}_{3}\right) \cdot{ }^{13} \mathrm{C}-$ NMR data (see Table 1). IR (KBr) $v_{\max } \mathrm{cm}^{-1}: 3334,2970,2854,1637,1581$, $1498,1377,1261 \mathrm{~cm}^{-1}$. UV $\left(\mathrm{CH}_{3} \mathrm{OH}\right) \lambda_{\max }(\log \varepsilon): 240$ (4.31), 274 (4.74), 323 (4.25), 377 (3.95). HR-EI-MS $m / z 408.1552[\mathrm{M}]^{+}$(Calcd for $\mathrm{C}_{24} \mathrm{H}_{24} \mathrm{O}_{6}$ : 408.1573). EI-MS $m / z 408\left([\mathrm{M}]^{+}\right.$, 64) 393 (100), 353 (29), 337 (14), 325 (17).

1,3,5-Trihydroxy-6', 6'-dimethyl-2H-pyrano $\left(2^{\prime}, 3^{\prime}: 6,7\right)$ xanthone (3): Yellow needles, mp, UV, EI-MS and ${ }^{1} \mathrm{H}-\mathrm{NMR}$ spectral data comparable with literature values. ${ }^{13} \mathrm{C}$-NMR data (see Table 1 ).

Dulxanthone A (4): Yellow powder, UV, IR, EI-MS, ${ }^{1} \mathrm{H}-$ and ${ }^{13} \mathrm{C}-\mathrm{NMR}$ spectral data comparable with literature values. ${ }^{13} \mathrm{C}$-NMR data (see Table 1).

1,5,6-Trihydroxy-3,7-dimethoxyxanthone (5): Pale brown powder, UV, EIMS and ${ }^{1} \mathrm{H}-\mathrm{NMR}$ spectral data comparable with literature values. ${ }^{13} \mathrm{C}-\mathrm{NMR}$ data (see Table 1).

Acknowledgements Prof. ShaoRong Guo, Institute of Medicinal Plant Development, Chinese Academy of Medical Sciences, Peking Union Medical College, China, is gratefully acknowledged for the plant identification. This work is supported by a grant from the National High Technology Research and Development Program of China (No.2002AA2Z343B).

\section{References}

1) Jena B. S., Jayaprakasha G. K., Sakarah K. K., J. Agric. Food. Chem., 50, 3431-3434 (2002)

2) Lee H. H., Lee H. K., Phytochemistry, 16, 2038-2040 (1977).

3) Likhitwitayawuid K., Phandungchareon T., Mahidol C., Ruchirawat S., Phytochemistry, 45, 1299-1301 (1997).

4) Likhitwitayawuid K., Phandungchareon T., Krungkrai J., Planta Med., 64, 70-72 (1998)

5) Wahyuni F. S., Byrne L. T., Dachriyanus, Dianita R., Jubahar J., Lajis N. H., Sargent M., Aust. J. Chem., 57, 223-236 (2004).

6) Mahabusarakam W., Chairerk P., Taylor W. C., Phytochemistry, 66, 1148-1153 (2005).

7) Moncache G. D., Botta B., J. Nat. Prod., 47, 620-625 (1984).

8) Ito C., Miyamoto Y., Nakayma M., Kawai Y., Rao K. S., Furukawa H., Chem. Pharm. Bull., 45, 1403-1413 (1997).

9) Ghosal S., Biswwas K., Chaudhuri R. K., J. Chem. Soc. Perkin Trans. I, 1977, 1597-1601 (1977).

10) Yin Z. Q., Ye W. C., Zhao S. X., J. Chin. Pharm. Univ., 33, 277-279 (2002).

11) Chaudhuri R. K., Ghosal S., Phytochemistry, 10, 2425-2432 (1971).

12) Jiang Y., Liu L., Tu P. F., Chin. J. Nat. Med., 3, 142-145 (2003).

13) Rukachaisirkul V., Ritthiwigrom T., Pinsa A., Sawangchote P., Taylor W. C., Phytochemistry, 64, 1149-1156 (2003). 